CORRIGENDUM

doi:10.1038/nature07826

\title{
Ecologically implausible carbon response?
}

Wim de Vries, Svein Solberg, Matthias Dobbertin, Hubert Sterba, Daniel Laubhann, Gert Jan Reinds, Gert-Jan Nabuurs, Per Gundersen $\&$ Mark A. Sutton

Nature 451, E1-E3 (2008)

In this Brief Communication Arising, the name of Daniel Laubhann was incorrectly listed as Daniel Laubhahn. 